\title{
Dataset for the reporting of urinary tract carcinoma-biopsy and transurethral resection specimen: recommendations from the International Collaboration on Cancer Reporting (ICCR)
}

\author{
M. Varma $\mathbb{D}^{1} \cdot$ J. R. Srigley ${ }^{2}$ F. Brimo ${ }^{3} \cdot$ E. Compérat ${ }^{4} \cdot$ B. Delahunt ${ }^{5} \cdot$ M. Koch ${ }^{6} \cdot$ A. Lopez-Beltran ${ }^{7} \cdot$ V. Reuter ${ }^{8}$. \\ H. Samaratunga ${ }^{9,10,11} \cdot$ J. H Shanks ${ }^{12} \cdot$ T. Tsuzuki $\mathbb{D}^{13} \cdot$ T. van der Kwast ${ }^{14} \cdot$ F. Webster ${ }^{15} \cdot$ D. Grignon ${ }^{16}$
}

Received: 7 June 2019 / Revised: 10 October 2019 / Accepted: 11 October 2019 / Published online: 4 November 2019

(c) The Author(s), under exclusive licence to United States \& Canadian Academy of Pathology 2019

\begin{abstract}
The International Collaboration on Cancer Reporting (ICCR) is an alliance of major pathology organisations in Australasia, Canada, Europe, United Kingdom, and United States of America that develops internationally standardised, evidence-based datasets for the pathology reporting of cancer specimens. This dataset was developed by a multidisciplinary panel of international experts based on previously published ICCR guidelines for the production of cancer datasets. It is composed of Required (core) and Recommended (noncore) elements identified on the basis of literature review and expert consensus. The document also includes an explanatory commentary explaining the rationale behind the categorization of individual data items and provides guidance on how these should be collected and reported. The dataset includes nine required and six recommended elements for the reporting of cancers of the urinary tract in biopsy and transurethral resection (TUR) specimens. The required elements include specimen site, operative procedure, histological tumor type, subtype/variant of urothelial carcinoma, tumor grade, extent of invasion, status of muscularis propria, noninvasive carcinoma, and lymphovascular invasion (LVI). The recommended elements include clinical information, block identification key, extent of $\mathrm{T} 1$ disease, associated epithelial lesions, coexistent pathology, and ancillary studies. The dataset provides a structured template for globally harmonized collection of pathology data required for management of patients diagnosed with cancer of the urinary tract in biopsy and TUR specimens. It is expected that this will facilitate international collaboration, reduce duplication of effort in updating current national/institutional datasets, and be particularly useful for countries that have not developed their own datasets.
\end{abstract}

M. Varma

wptmv@cf.ac.uk

1 Department of Cellular Pathology, University Hospital of Wales, Cardiff, UK

2 Department of Laboratory Medicine and Pathobiology, University of Toronto, Toronto, ON, Canada

3 Department of Pathology, McGill University Health Center, Montréal, QC, Canada

4 Department of Pathology, Hopital Tenon, HUEP, Sorbonne University, Paris, France

5 Department of Pathology and Molecular Medicine, Wellington School of Medicine and Health Sciences, University of Otago, Wellington, New Zealand

6 Department of Urology, Indiana University School of Medicine, Indianapolis, IN, USA

7 Department of Pathology, Champalimaud Clinical Center, Lisbon, Portugal
8 Department of Pathology, Memorial Sloan Kettering Cancer Center, New York, NY, USA

9 Aquesta Specialized Uropathology, Brisbane, QLD, Australia

10 The University of Queensland, Centre for Clinical Research, Brisbane, QLD, Australia

11 Princess Alexandra Hospital, Brisbane, QLD, Australia

12 Department of Histopathology, The Christie NHS Foundation Trust, Manchester, UK

13 Department of Pathology, Aichi Medical University, Aichi, Japan

14 Laboratory Medicine Program, University Health Network, University of Toronto, Toronto, ON, Canada

15 International Collaboration on Cancer Reporting, Sydney, NSW, Australia

16 Department of Pathology and Laboratory Medicine, Indiana University School of Medicine, IUH Pathology Laboratory, Indianapolis, IN, USA 


\section{Introduction}

Structured reporting protocols facilitate pathology reporting of cancer specimens by ensuring inclusion of all clinically relevant information in a user-friendly format thereby aiding use of data for patient management, epidemiology, audit, and research. Datasets or checklists for pathology reporting have been independently developed by several organisations across the world including the Royal College of Pathologists, United Kingdom (RCPath) [1], the College of American Pathologists, USA (CAP) [2], and Royal Australasian College of Pathologists (RCPA) [3]. Although these protocols are broadly similar, there are significant differences in content and terminology that could hinder international comparison and research. Hence in 2011, the CAP, RCPath, RCPA, and the Canadian Association of Pathologists in association with the Canadian Partnership Against Cancer (Canada) formed the International Collaboration on Cancer Reporting (ICCR) and successfully piloted the development of datasets for pathology reporting of cancers of the prostate (radical prostatectomy), endometrium, melanoma, and lung cancers [4-7]. This quadripartite collaboration together with the European Society of Pathology was incorporated in 2014 as a not-for-profit organisation that was joined by the American Society of Clinical Pathology and the Faculty of Pathology, Royal College of Physicians of Ireland. The ICCR has developed strategic alliances with other international cancer organisations such as the International Agency for Research on Cancer (IARC), Union for International Cancer Control (UICC), and American Joint Committee on Cancer (AJCC). The ICCR coordinates the production of evidence-based international pathology reporting datasets developed by a panel of internationally recognised expert pathologists and clinicians that are published in peer-reviewed journals [811]. The datasets have a consistent style and content, and contain all the parameters needed to guide management and prognostication for individual cancers. The datasets are freely available from the ICCR website http://www.iccr-ca ncer.org.

The World Health Organization (WHO) Classification of Tumours 'Blue books' is an integral part of all ICCR datasets and in 2013 the ICCR committed to synchronise its schedule of dataset development with the publication of the WHO blue book series. Thus, following the publication of the 4th edition of the WHO Classification of Tumours of the Urinary System and Male Genital Organs in 2016 [12], the ICCR initiated the development of genitourinary datasets to align with this publication. Included in this suite are four datasets providing guidance for the structured reporting of pathology data for cancers of the urinary tract [13]. The main points in the dataset for reporting of cancer in biopsy and TUR specimens of the urinary tract are summarised in this paper. The reader is referred to the ICCR website for a more comprehensive discussion [14].

\section{Methods}

This dataset was developed on the basis of the guidelines agreed by the Dataset Steering Committee (DSC) of the ICCR [15]. The DSC appointed a Chair (DG) to develop datasets for the reporting of cancers of the urinary tract and together they identified ten other expert urological pathologists who together with the Chair, a urologist, an ICCR representative (JS), and a Project Manager (FW) formed the Dataset Authoring Committee (DAC). The expert panel included two pathologists each from the USA (DG, VR), UK (JSh, MV), Europe (ALB, EC), Canada (FB, TvK), and Australasia (BD, HS) together with a pathologist from Japan (TT) and a urologist from the USA (MK). The ICCR representative provided guidance and support to the Chair of the DAC regarding ICCR standards and ensured harmonization across datasets, while the Project Manager coordinated the development process.

In line with other ICCR datasets, this dataset for reporting of cancers in biopsy and TUR specimens from the urinary tract included a set of elements and value lists (responses) accompanied by a commentary. The elements were categorized as either required or recommended. Required (core) elements were those unanimously agreed by the expert panel to be essential for diagnosis, prognostication, and/or patient management and generally required evidentiary support at Level III-2 or above [based on prognostic factors in the National Health and Medical Research Council (NHMRC) levels of evidence document, and defined as 'Analysis of prognostic factors amongst persons in a single arm of a randomized controlled trial'] [16]. Specific levels of evidence were not assigned to each required or recommended data item. All tumor subtypes defined in 2016 WHO classification [12] were categorized as required. Recommended (noncore) elements were those that did not meet the above standard but were considered by the panel to be clinically important or representing good practice. The commentary clarified the elements, explained the rationale behind the above categorization of individual data items, and cited published evidence that supported its inclusion.

The initial working draft of this dataset was developed by the Project Manager following a review of all published cancer datasets pertaining to carcinoma of the urinary tract and reporting guidelines published in review articles or other international websites. Following editing by the Chair, this draft was circulated to the DAC and discussed in a series of teleconferences. The Chair then reedited the documents based on these discussions and recirculated them 
to the members of the DAC for further review and discussion in a series of email communications until consensus was achieved. The finally approved document was formatted and uploaded on the ICCR website for a period of 2 months for public comment. The documents were reviewed in the light of the feedback, approved by the DAC, and finally ratified by the DSC.

This DAC developed four datasets to facilitate reporting of cancers of the urinary tract. This dataset deals with biopsy and TUR specimens. Other datasets were created for urethrectomy, cystectomy/cystoprostatectomy/diverticulectomy, and nephrourethrectomy/ureterectomy specimens. These may be accessed at http://www.iccr-cancer.org/data sets/published-datasets/urinary-male-genital.

\section{Results}

This dataset deals with reporting of primary carcinomas (noninvasive and invasive) in biopsy and TUR specimens of the bladder, urethra, ureter, and renal pelvis. It should not be used for papillomas and papillary urothelial neoplasms of low malignant potential as these are not carcinomas. Separate datasets should be completed for tumors in different locations. Tumours arising in the most distal portion of the penile urethra should be reported using the penile and distal urethral carcinoma dataset [17].

The required and recommended data items in this dataset are summarised in Table 1.

\section{Clinical information}

Knowledge of any relevant history is critical for accurate diagnosis [18-21] but this element is categorised as a recommended rather than a required item as it is the responsibility of the clinician to provide information that will have an impact on the diagnostic process or affect its interpretation.

Table 1 Required and recommended elements for the pathology reporting of urinary tract carcinoma-biopsy and transurethral resection specimens

\begin{tabular}{ll}
\hline Required & Recommended \\
\hline Specimen site & Clinical information \\
Operative procedure & Block identification key \\
Histological tumour type & Substaging T1 disease \\
Histological subtype/variant & Associated epithelial lesions \\
Histological tumour grade & Coexistent pathology \\
Extent of invasion & Ancillary studies \\
Status of muscularis propria & \\
Lymphovascular invasion & \\
Non-invasive carcinoma & \\
\hline
\end{tabular}

The pathologist must be informed about any history of urothelial neoplasia and particularly about therapies such as Bacillus Calmette-Guerin (BCG) and mitomycin C, which could result in histological changes with the potential for misdiagnosis [22, 23]. Pelvic radiation therapy may lead to reactive epithelial proliferations that very closely mimic invasive carcinoma [24, 25]. Prior surgical procedures such as TUR and biopsy may be associated with nephrogenic adenoma that could mimic recurrent tumor clinically and pathologically $[26,27]$.

Knowledge of the cystoscopic findings could inform the interpretation of a bladder biopsy. For example, examination of deeper levels would be indicated if a papillary lesion observed at cystoscopy is not seen in the tissue levels examined.

Finally, knowledge of a history of carcinoma elsewhere such as prostatic adenocarcinoma, colorectal adenocarcinoma, or cervical squamous cell carcinoma may help the interpretation of biopsy/TUR specimens.

\section{Specimen site}

Urothelial neoplasia represents a field change across the entire urinary tract so development of synchronous or metachronous tumors in different parts of the urinary tract is not uncommon. Hence, it is required to record the site of origin of a biopsy/ TUR specimen. If biopsies are from different locations (bladder, ureter, urethra, renal, and pelvis), then a separate dataset should be completed for each specimen site.

The specific anatomic site also informs histological interpretation as differential diagnosis and key staging landmarks varies with the site of origin of a biopsy/TUR specimens, including its location within individual sites. The possibility of urachal lesions would have to be considered in specimens from the dome/anterior wall of the urinary bladder, while secondary tumors from adjacent organs such as uterus and prostate would be part of the differential diagnosis in specimens from the bladder neck and trigone region. The distribution of muscularis mucosae (MM) fibres varies by location in the urinary bladder and so knowledge of location can assist in evaluation of smooth muscle in the context of staging parameters [28].

\section{Operative procedure}

Documentation of the specific procedure performed is a standard part of any pathology report and categorised as a required data item in this dataset.

\section{Block identification key}

Knowledge of the origin/designation of each tissue block aids microscopic interpretation and its inclusion in the 
pathology report is particularly important if there is a need for internal or external review. Recording the origin/designation of tissue blocks also facilitates retrieval of blocks for further immunohistochemical or molecular analysis, research studies, or clinical trials. Although block identification is not a required element within the synoptic report, we would consider it required within the report text (generally included in the gross description section).

\section{Histological tumor type}

Recording the histological tumor type using the 2016 WHO classification [12] is a required element of this dataset as this parameter is often of prognostic and therapeutic significance (Table 2). As in the 2004 WHO classification [29], a tumor is classified as a urothelial carcinoma if there is any urothelial differentiation [including associated urothelial carcinoma in situ (CIS)] with any other types present reported with an estimated percentage. Thus, a carcinoma showing 20\% urothelial differentiation and $80 \%$ glandular differentiation would be reported under the histological tumor type Urothelial carcinoma and then under histological subtype/variant as glandular (80\%). An exception to this rule is for cases with any amount of neuroendocrine component (small cell neuroendocrine carcinoma or large cell neuroendocrine carcinoma) where classification is now in the neuroendocrine tumor category. Thus, a mixed tumor with 30\% small cell neuroendocrine carcinoma and $70 \%$ urothelial carcinoma would be reported under the histological tumor type as neuroendocrine tumor (small cell neuroendocrine carcinoma) and then under histological tumor type-other, specify-urothelial carcinoma (70\%). This is a controversial issue as reflected by the different approaches recommended by WHO 2016 in chapters on the neuroendocrine tumors and urothelial carcinoma variants. For a diagnosis of small cell carcinoma, the former required the small cell component to constitute the majority of the tumor but the variants chapter indicated that tumors with any small cell component should be categorised as such. ICCR recommends the latter approach but recognises that the percentage of the neuroendocrine component could inform patient management, particularly with newer treatment modalities such as immunotherapy.

Biopsies and TURs that contain pure adenocarcinoma or pure squamous cell carcinoma should be diagnosed as such but with a comment that it is not possible to definitively exclude the possibility of a urothelial carcinoma with extensive glandular or squamous differentiation without examination of the entire tumor and background flat epithelium. There are no reliable immunohistochemical markers to distinguish these possibilities with certainty in an individual case [30]. The presence of keratinizing squamous metaplasia or intestinal metaplasia, particularly when
Table 2 World Health Organization (WHO) Classification of tumours

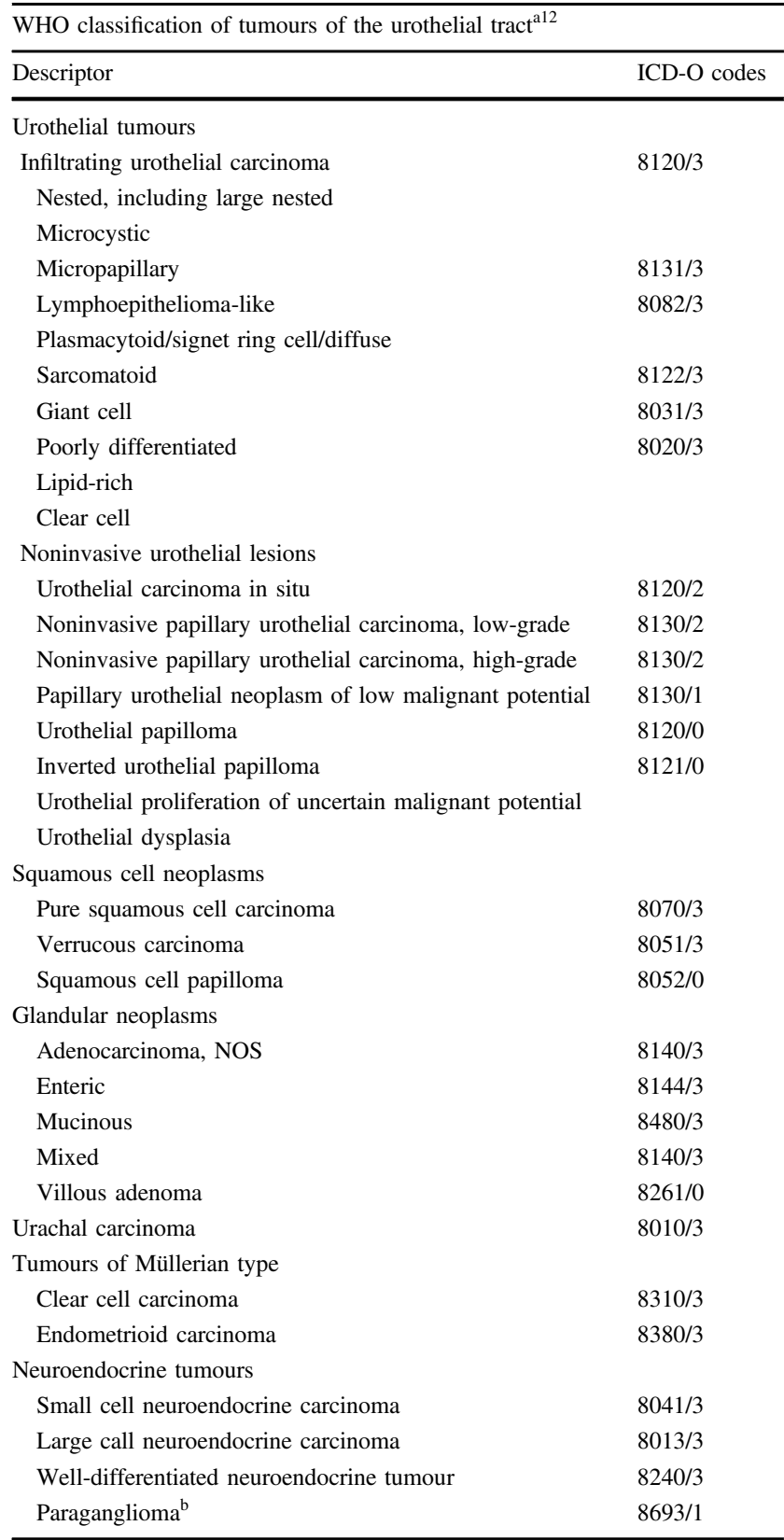

Pathology and genetics of the urinary system and male genital organs ${ }^{a}$ The morphology codes are from the International Classification of Diseases for Oncology (ICD-O). Behaviour is coded $/ 0$ for benign tumours; / 1 for unspecified, borderline, or uncertain behaviour; $/ 2$ for carcinoma in situ and grade III intraepithelial neoplasia; and /3 for malignant tumours

${ }^{\mathrm{b}}$ Paraganglioma is not an epithelial derived tumour

${ }^{\circ}$ Reproduced with permission from WHO/International Agency for Research on Cancer (IARC)

associated with dysplasia could support a diagnosis of primary squamous cell carcinoma [31] or adenocarcinoma [32], respectively, but a definitive diagnosis of either should be made with caution in biopsy or TUR material. Diagnosis 
of primary adenocarcinoma or squamous cell carcinoma of the urinary bladder also requires clinical correlation to exclude the possibility of origin from extravesical sites such as the rectum and cervix.

The 2016 WHO classification includes urachal carcinomas as a separate category but diagnosis of this subtype requires clinicopathologic correlation with diagnostic criteria including location in the bladder dome or anterior wall, an epicentre in the bladder wall or perivesical tissue, the absence of diffuse cystitis glandularis/intestinal metaplasia outside of the dome/anterior wall region and the absence of a known primary elsewhere [12, 33]. If a diagnosis of urachal carcinoma is rendered the histologic type should be specified. Although the majority (over 80\%) of urachal carcinomas are adenocarcinoma, other subtypes such as urothelial carcinoma, squamous cell carcinoma, and small cell neuroendocrine carcinoma may arise in the urachal region. The WHO also recognises a category of "mucinous cystic tumor of low malignant potential" that cannot be diagnosed with certainty in biopsy/TUR specimens [12]. There are no reliable immunohistochemical markers to distinguish adenocarcinomas of urachal origin from primary adenocarcinomas of the bladder proper or from secondary adenocarcinomas of gastrointestinal origin [30, 33, 34].

Also new in the 2016 WHO classification of bladder cancers is the category of Müllerian tumors that consists primarily of clear cell adenocarcinoma and rare examples of endometrioid carcinoma, which may arise from endometriosis or rarely Müllerianosis [12, 35-37]. These tumors are morphologically and immunophenotypically similar to their counterparts in the female genital tract and hence diagnosis of a primary Müllerian bladder tumor requires clinical exclusion of an extravesical primary [38]. Clear cell adenocarcinoma must also be distinguished from urothelial carcinoma with divergent differentiation along Müllerian lines that would be classified under urothelial carcinoma [39]. Expression of urothelial immunomarkers (such as GATA3 and uroplakin III) or associated urothelial component would suggest the latter possibility [40].

The neuroendocrine tumor category includes small cell neuroendocrine carcinoma, large cell neuroendocrine carcinoma, well-differentiated neuroendocrine tumor, and paraganglioma with small cell neuroendocrine carcinoma being by far the most common [12]. About half of these tumors are admixed with another component, most commonly urothelial carcinoma (invasive and/or in-situ). Mixed tumors with any small cell neuroendocrine carcinoma component are generally managed as small cell neuroendocrine carcinoma [41] and hence carcinomas with any small cell neuroendocrine component are categorised as neuroendocrine subtype in this dataset. The diagnosis is defined by morphologic criteria but most cases do show immunohistochemical evidence of neuroendocrine differentiation. The most sensitive immunohistochemical markers are CD56 and synaptophysin, although the former is less specific [30]. TTF-1 is expressed in about $50 \%$ of cases and hence cannot be used to identify the site of origin of a tumor [42]. In cases with pure small cell morphology the possibility of direct spread from an adjacent organ or metastasis must be excluded clinically.

Lastly there are carcinomas arising in the urinary bladder that have no specific differentiation and based on exclusion of metastasis from another site are considered to be primary in the urinary tract. In the 2004 WHO classification these were included as a variant of urothelial carcinoma [29] but given that by definition they have no urothelial differentiation these should be reported using the "carcinoma, type cannot be determined" category.

\section{Histologic subtype/variant}

Several morphologic variants are recognised in the WHO 2016 classification of bladder tumors (Table 2) [12]. Some of these have important prognostic or therapeutic implications, while recognition of other variant tumor morphology permits distinction from other histological mimics. For example, in T1 urothelial carcinoma, the presence of variant histology is one feature that is used in determining whether to consider immediate cystectomy [41, 43], while the nested variant urothelial carcinoma may be very difficult to distinguish from von Brunns nests, particularly in superficial biopsies.

The level of evidence for specific variants having independent prognostic information varies from the variant having no clinical significance but being important diagnostically (e.g., nested, microcystic, etc), to no data, to data indicating the variant has prognostic significance (e.g., micropapillary, plasmacytoid, and sarcomatoid). Rather than making reporting of specific subtypes that have some supporting data mandatory and others lacking data recommended, this dataset has opted to make the entire category a required element.

In concordance with the WHO 2016 monograph [12], this ICCR dataset requires the reporting of the percentage of each variant histology and divergent differentiation (glandular or squamous), when present. However, the data supporting this is very limited and is only available for some variants such as micropapillary, sarcomatoid, and lymphoepithelioma-like. There is also uncertainty regarding the reproducibility of variant percentage estimation and the amounts of each variant that would be clinically significant.

\section{Histological tumor grade}

Histological tumor grade is a critical parameter in the management of noninvasive papillary tumors. ICCR 
recommends the 2016 WHO classification system [12] that was proposed by the International Society of Urological Pathology (ISUP) in 1997 [44] and adopted by the WHO in 2004 [29]. This system is now recommended by almost all major pathology and urology organizations as the preferred grading system $[19,21]$.

The 2016 WHO classification for noninvasive papillary urothelial tumors [12] is a three-tiered system with the lowest category of papillary urothelial neoplasm of low malignant potential (PUNLMP) considered to be a benign neoplasm [45] and hence not reported using this dataset. The other two categories, low-grade and high-grade papillary urothelial carcinoma differ significantly in the risk of progression to invasive carcinoma and death from bladder cancer [45]. Hence, the grade of noninvasive papillary carcinoma is the major parameter used to guide the choice of therapy in these patients [43]. Other features of importance in predicting outcome of patients with Ta papillary tumors are number of tumors/multifocality, tumor size, the presence of associated CIS, and a history of prior recurrence [46]. It has also been suggested that for low grade papillary tumors the frequency and duration of cystoscopic follow up can be reduced [47, 48].

A major rationale of the ISUP 1997/WHO 2004/WHO 2016 grading system was to address the issue of a large, heterogenous intermediate (grade 2) category in WHO 1973 grading system [44]. There has been some concern about the heterogeneity of the high-grade category in the ISUP 1997/WHO 2004/WHO 2016 grading system [49] and some published guidelines mandate the reporting of both the current WHO grade with the 1973 grade [43, 50]. Such an approach could identify patients at lower end of highgrade (high-grade/grade 2) who may not need aggressive therapy such as cystectomy for post-BCG therapy highgrade recurrence.

This ICCR dataset follows the WHO 2016 approach and recommends reporting of the WHO 2016 grade as a required element and the inclusion of other grading systems as optional [12].

The grading of $\mathrm{T} 1$ invasive urothelial carcinoma is another area of controversy. In studies using the 1998 ISUP/ WHO 2004 grading system the vast majority of such tumors were high grade [51] and the International Consultation on Urologic Disease (ICUD) pathology group concluded that all invasive carcinomas should be considered high grade [18]. It has been noted that there are variants of urothelial carcinoma that have low grade cytologic features such as the nested variant, but these appear to behave stage for stage like usual high grade carcinoma [52]. When variant histology such as this is present, it is recommended that the tumors should be reported as high grade despite the bland cytology in order to reflect the biologic behaviour [53]. Small series of invasive papillary urothelial carcinoma, in which both the papillary and invasive components were low-grade have also been reported [54]. Nonetheless it is equally apparent that many pathologists have graded invasive urothelial carcinomas using the 1973 WHO and other systems and have demonstrated its prognostic significance $[43,55,56]$. ICCR endorses the 2016 WHO recommendation to grade invasive carcinoma using the WHO 2004 system recognising that the vast majority of tumors will be high grade [12]. If invasive tumors are graded using an alternative grading system this should be indicated.

There is general consensus that tumor grade is of little clinical significance in carcinomas that invade the muscularis propria.

A final area of controversy in noninvasive papillary urothelial carcinoma grading relates to grade heterogeneity that has been reported in up to $32 \%$ of cases $[57,58]$. It is currently recommended that tumor grade be assigned based on the highest grade present but some authors have recommended considering a tumor low grade if the high grade component accounts for $<5 \%$ of the tumor volume [57]. Some studies suggest that tumors with a limited amount of high-grade component have a better outcome than cases that are purely or predominantly high-grade [5759]. The limited data does not allow for a definitive statement regarding reporting of cases with a small volume of high grade tumor or to determine what percentage of high grade tumor is necessary to indicate a significantly worse prognosis. ICUD recommended against the application of an arbitrary percentage of high grade tumor when assigning grade [18]. The 2016 WHO recommends grading based on the highest grade component and acknowledges the uncertainty of how to approach cases with a small proportion of high grade tumor [12]. It does indicate that "it may be prudent to state the proportion of high-grade disease." ICCR concurs with WHO 2016 and recommends reporting the highest grade but including a comment in cases where the high-grade component is estimated to be $<10 \%$.

\section{Extent of invasion}

Reporting the extent of invasion is a critical part of the assessment of carcinomas arising in the urinary tract. The elements included reflect the anatomic landmarks that are essential to the pathologic staging of each tumor and vary by site within the urinary tract [60]. It is not appropriate to assign pathologic stage on biopsy or TUR specimens and pathologic stage is not an element within this dataset. It is however possible, based on the assessment of the extent of invasion to recognise the least pathological stage possible in a given case. The stage reported in a transurethral resection of bladder tumors (TURBT) specimen would be part of the clinical stage (e.g., T2 rather than pT2).

The diagnosis of invasion can be challenging as documented by several studies [61-63]. Studies have also 
demonstrated lack of agreement even among pathologists with special interest in urologic pathology [64]. Following the principles of the AJCC TNM staging system the diagnosis of invasion should be limited to cases with unequivocal invasion [60].

Identification of invasion of smooth muscle fibres in specimens from the renal pelvis, ureter, and urethra all indicate $\mathrm{T} 2$ disease. In the urinary bladder the presence of the MM complicates the interpretation as involvement of these fibres still represents a T1 tumor [65]. Unlike in the colon, MM is an incomplete layer in the bladder and may be absent in bladder biopsy and TUR specimens [28]. Similarly, the lamina propria is of variable thickness in the bladder and muscularis propria is very superficial in the trigone/bladder neck region, where from a practical perspective involvement of smooth muscle essentially always indicates muscularis propria invasion. The $\mathrm{MM}$ is associated with a vascular plexus that can be used as a surrogate to mark the plane of the MM when the smooth muscle bundles of this layer are not represented in the specimen. MM fibres are typically thin and wispy forming small bundles that taper at the ends and usually are only a few cells thick but may occasionally be thickened and better defined, more closely mimicking muscularis propria. Smoothelin, a cytoskeletal protein is differentially expressed in the muscularis propria and not the MM [66]. Application in challenging cases can be helpful but for the most part the marker has not gained widespread application and is not currently recommended routinely for subclassifying muscle type $[30,67,68]$. When there is uncertainty whether the smooth muscle involvement represents MM or muscularis propria this should be specifically commented upon and a repeat TUR may be necessary to determine the true depth of involvement [68].

Urothelial carcinoma in the prostatic urethra is generally seen in association with a bladder tumor [69-71]. Involvement is usually by urothelial CIS but occasionally papillary tumors are seen. Extension into the prostatic ducts is frequently present in these cases and should not be mistaken for invasion. Immunohistochemistry is frequently required to distinguish urothelial carcinoma from high grade prostatic adenocarcinoma [30].

\section{Substaging T1 disease}

There have been many efforts to risk stratify $\mathrm{T} 1$ bladder cancers by substaging them based on depth of invasion using either anatomical or quantitative criteria.

The largest volume of literature has tried to use the MM as a landmark to subdivide $\mathrm{T} 1$ tumors into two (T1a: superficial to MM, T1b: involving or deep to MM) or three (T1a: superficial to MM, T1b: involving MM, T1c: deep to MM) subgroups. These studies have shown that deeper invasion is associated with a worse progression free and cancer specific survival [72-76].

The other approach to $\mathrm{T} 1$ substaging involves quantitation of the extent of invasion either as maximum depth of invasion perpendicular to the mucosal surface $[77,78]$ or the maximum linear length of the invasive tumor, irrespective of the orientation [75, 78-82]. These methods have also shown extent of $\mathrm{T} 1$ invasion to correlate with recurrence free-, progression free-, and cancer specific-survival. However, there is no consensus on cut-off points for substratifying $\mathrm{T} 1$ disease and each of these has limitations such as the establishment of cut points and specimen orientation [75, 77-82].

Recent guidelines such as the ICUD recommendations, 2016 WHO, and the CAP reporting guidelines recommend that pathologists provide some indication of extent of lamina propria invasion (e.g., focal, multifocal, extensive, etc or involvement of MM) without specifying a preferred method [12, 21].

Because of the potential for additional information in T1 tumors to directly impact clinical decision making the ICCR guidelines have included substaging of TI disease as a nonmandatory recommended element. The dataset also provides for alternative methods for reporting as there is insufficient data to recommend one alternative over the others.

\section{Status of muscularis propria}

Documentation of the presence or absence of muscularis propria is important to determine the adequacy of a biopsy or TUR specimen that contains an invasive carcinoma $[19,43,83]$. For such patients, the absence of muscularis propria in a TURBT would suggest that the procedure was too superficial and be an indication for a repeat TUR if treatment is other than cystectomy. Absence of muscularis propria in a TURBT specimen has been shown to be associated with a significantly increased risk of residual disease and early recurrence [84]. Presence or absence of muscularis propria in a biopsy or TUR specimen, irrespective of the presence or absence of invasive carcinoma and is therefore a required data item in this dataset.

\section{Lymphovascular invasion}

Several studies including a very large multi-institutional series and a phase 3 clinical trial have found Lymphovascular invasion (LVI) in urothelial carcinoma in radical cystectomy specimens and resection specimens for upper tract urothelial carcinoma to be a highly significant predictor of outcome [85-93].

In contrast, there is less data regarding the significance of LVI in bladder biopsy or TURBT material and these have 
almost all been based on H\&E evaluation with limited utilisation of immunohistochemistry [78, 94-103]. Although they report conflicting results, majority of the studies found LVI to be an independent predictor of outcome. Specific data on LVI determination in biopsy/TUR specimens of upper tract and urethra are not available.

The frequency of identification of LVI on bladder biopsy or TURBT material has ranged from $<10 \%$ to as high as $67 \%$. The problem with recognising LVI by urothelial carcinoma on H\&E sections have been discussed by Algaba [104] and Lopez-Beltran et al. [83] and the importance of utilising strict criteria emphasised. The possibility of routinely performing immunohistochemistry on $\mathrm{T} 1$ cases has been discussed but with little data. The ICUD pathology committee concluded that they could not recommend the general use of immunohistochemistry in the routine setting for identification of LVI in bladder cancer [53].

Although the data on LVI in biopsy/TUR specimens is limited, the compelling evidence in large resection studies of urothelial carcinoma of the urinary bladder and upper tract support its inclusion as a required element in this dataset.

\section{Noninvasive carcinoma}

Noninvasive papillary tumors account for 70-75\% of newly diagnosed cases with over one-half being in the lower grade categories (papillary urothelial neoplasm of low malignant potential, low grade papillary carcinoma) [105, 106]. Urothelial CIS often coexists with high-grade papillary urothelial carcinoma and is found in association with invasive urothelial carcinoma in up to $65 \%$ of cases [107-109]. CIS in a pure form without associated papillary tumor is rarely encountered, accounting for only 1-3\% of newly diagnosed urothelial tumors [107].

The identification of CIS coexisting with papillary carcinoma has significance for prognosis and treatment. Patients with low-grade noninvasive urothelial carcinoma would be treated with BCG if there is associated CIS, which is by definition high-grade. CIS and noninvasive papillary carcinoma develop by different genetic pathways and have different biologic behaviour and so are considered as different entities within the noninvasive category [110].

Recognition of CIS associated with high-grade noninvasive papillary carcinoma is straight-forward when the papillary tumor and the CIS are present in different tissue fragments or in specimens submitted from different sites. However, when severely atypical flat lesion is present adjacent to and in continuity with a high-grade papillary tumor it can be difficult to decide whether the flat area represents a "shoulder" of the papillary tumor or coexisting CIS. There are no generally accepted criteria for making this clinically significant decision. We recommend making the diagnosis of associated CIS in this situation if there is a gap of normal urothelium between the papillary tumor and the flat lesion or if the morphology of the flat lesion is different than that of the epithelium on the surface of papillary fronds.

For patients with T1 disease the presence of CIS indicates a significantly increased risk of subsequent recurrence and of progression to $\mathrm{T} 2$ disease. The presence of associated CIS in newly diagnosed high grade $\mathrm{T} 1$ disease may also be used to support early cystectomy [43, 111]. For patients presenting with invasive urothelial carcinoma there are data that such cases arising through the "papillary" pathway have stage for stage a better prognosis than those developing via the "flat" pathway [112, 113].

For patients presenting with invasive urothelial carcinoma, the recognition and documentation of an associated noninvasive papillary carcinoma and/or CIS remains important and hence this is categorised as a required data item.

There is also evidence that the extensiveness of the CIS is significant and so distinguishing between a single focus and diffuse (or multifocal) disease is important. Multifocality of CIS is easy to recognise when present in separately submitted biopsies from different sites but difficult when encountered in a TURBT specimen. For the purpose of this dataset, the presence of CIS involving more than one tissue fragment in a TURBT specimen is categorised as multifocal.

CIS of the urinary tract is generally of urothelial type but nonurothelial CIS can also occur in these sites. Most frequently this is squamous cell CIS typically in association with keratinizing squamous metaplasia. This can be identified in patients with invasive squamous cell carcinoma but may be encountered in the absence of invasive disease. Adenocarcinoma in situ is not a well-defined lesion in the urinary tract. In cases of intestinal metaplasia varying degrees of atypia can be seen up to high grade dysplasia, a term we would prefer rather than adenocarcinoma in situ. Squamous or glandular differentiation in urothelial CIS should not be misinterpreted as squamous or adenocarcinoma in situ respectively.

\section{Coexistent pathology}

Biopsy and endoscopic resection specimens from throughout the urinary tract that are diagnosed with carcinoma can also show a number of nonneoplastic conditions. Although some findings such as keratinizing squamous metaplasia and diffuse intestinal metaplasia may be relevant in a specific case the reporting of these findings does not have sufficient significance to be considered a required element. Hence it is categorised as a recommended data item in this dataset. 


\section{Associated epithelial lesions}

A variety of neoplastic lesions that fall short of carcinoma are recognised in the urinary tract. These include papillary lesions such as urothelial papilloma, PUNLMP, and inverted urothelial papilloma. Similarly, flat lesions such as urothelial proliferation of uncertain malignant potential, urothelial dysplasia, keratinizing squamous metaplasia with dysplasia, and intestinal metaplasia with dysplasia can be seen. Identification of these may have diagnostic implications (e.g., the presence of keratinizing squamous metaplasia with dysplasia supporting the diagnosis of primary squamous cell carcinoma) but do not have known proven prognostic or clinical significance otherwise. While for completeness it may be useful to report such findings, it is not considered to be a required element in the context of a carcinoma diagnosis and hence categorised as recommended.

\section{Ancillary studies}

Currently there are no ancillary studies that are recommended for routine use in urothelial carcinoma of the urinary tract and hence this is not a required data item. If immunohistochemical studies are performed for resolving differential diagnosis, to assist in staging, aid the detection of LVI, or predict response to treatment (e.g., PD-L1), they could be listed in this section. If other ancillary studies are performed these should also be included in the report.

\section{Discussion}

Histopathologic data are critical in the management of patients with carcinomas of the urinary tract. Hence, it is imperative that pathologists provide accurate and comprehensive data in biopsies and TUR specimens, particularly in patients with clinically noninvasive or superficially invasive carcinomas of the urinary tract. Adoption of structured (synoptic) reporting protocols have been shown to improve completeness of histopathology data in several organs [114]. Structured reports are also more user-friendly and facilitate electronic transfer of data to cancer registries and other end users of pathology data.

Several national organisations and institutions have developed structured reports, but these show significant variation hindering international data comparison and benchmarking. Moreover, structured site-specific pathology reports are cumbersome to update while other countries may lack the resources to produce such documents. The ICCR datasets that have been developed by an international panel of experts to meet global needs can facilitate timely revision and adoption in the light of advancing insights and emerging predictive biomarkers. This ICCR dataset and accompanying commentary can be used as a framework by organisations developing datasets for pathology reporting of cancers of the urinary tract. The required elements in this dataset represents the minimum requirements for pathology reporting and individual pathologists or organisations may elect to collect additional data or perform more extensive special tests than is recommended by this dataset.

In summary, this dataset provides a structured template for globally harmonised collection of pathology data required for management of patients diagnosed with cancer of the urinary tract in biopsy and TUR specimens. It is expected that this will facilitate international collaboration, reduce duplication of effort in updating current national/ institutional datasets and be particularly useful for countries that have not developed their own datasets.

\section{Compliance with ethical standards}

Conflict of interest The authors declare that they have no conflict of interest.

Publisher's note Springer Nature remains neutral with regard to jurisdictional claims in published maps and institutional affiliations.

\section{References}

1. Royal College of Pathologists UK. Cancer datasets and tissue pathways. https://www.rcpath.org/profession/guidelines/cancerdatasets-and-tissue-pathways.html. (2019). Accessed 03 May 2019.

2. College of American Pathologists. Cancer protocol templates. http://www.cap.org/web/oracle/webcenter/portalapp/pagehierarchy/ cancer_protocol_templates.jspx?_afrLoop $=212779956262482 \# ! \%$ 40\%40\%3F_afrLoop\%3D212779956262482\%26_adf.ctrl-state\% 3Da98ka87ex_4 (2019). Accessed 03 May 2019.

3. Royal College of Pathologists of Australia. Cancer protocols. https://www.rcpa.edu.au/Library/Practising-Pathology/StructuredPathology-Reporting-of-Cancer/Cancer-Protocols (2019). Accessed 03 May 2019.

4. Kench JG, Delahunt B, Griffiths DF, Humphrey PA, McGowan $\mathrm{T}$, Trpkov K, et al. Dataset for reporting of prostate carcinoma in radical prostatectomy specimens: recommendations from the International Collaboration on Cancer Reporting. Histopathology. 2013;62:203-18.

5. McCluggage WG, Colgan T, Duggan M, Hacker NF, Mulvany N, Otis C, et al. Dataset for reporting of endometrial carcinomas: recommendations from the International Collaboration on Cancer Reporting (ICCR) between United Kingdom, United States, Canada, and Australasia. Int J Gynecol Pathol. 2013;32:45-65.

6. Scolyer RA, Judge MJ, Evans A, Frishberg DP, Prieto VG, Thompson JF, et al. Dataset for pathology reporting of cutaneous invasive melanoma: recommendations from the International Collaboration on Cancer Reporting (ICCR). Am J Surg Pathol. 2013;37:1797-814.

7. Jones KD, Churg A, Henderson DW, Hwang DM, Ma Wyatt J, Nicholson AG, et al. Dataset for reporting of lung carcinomas: recommendations from International Collaboration on Cancer Reporting. Arch Pathol Lab Med. 2013;137:1054-62. 
8. McCluggage WG, Judge MJ, Clarke BA, Davidson B, Gilks CB, Hollema H, et al. Dataset for reporting of ovary, fallopian tube and primary peritoneal carcinoma: recommendations from the International Collaboration on Cancer Reporting (ICCR). Mod Pathol. 2015;28:1101-22.

9. Churg A, Attanoos R, Borczuk AC, Chirieac LR, Galateau-Salle F, Gibbs A, et al. Dataset for reporting of malignant mesothelioma of the pleura or peritoneum: recommendations from the International Collaboration on Cancer Reporting (ICCR). Arch Pathol Lab Med. 2016;140:1104-10.

10. Nicholson AG, Detterbeck F, Marx A, Roden AC, Marchevsky AM, Mukai K, et al. Dataset for reporting of thymic epithelial tumours: recommendations from the International Collaboration on Cancer Reporting (ICCR). Histopathology. 2017;70:522-38.

11. McCluggage WG, Judge MJ, Alvarado-Cabrero I, Duggan MA, Horn LC, Hui P, et al. Dataset for the reporting of carcinomas of the cervix: recommendations from the International Collaboration on Cancer Reporting (ICCR). Int J Gynecol Pathol. 2018;37:205-28.

12. Moch H, Humphrey PA, Ulbright TM, Reuter RE. WHO classification of tumours of the urinary system and male genital organs. Lyon, France: IARC Press; 2016.

13. International Collaboration on Cancer Reporting. Urinary/male genital cancer datasets. http://www.iccr-cancer.org/datasets/ published-datasets/urinary-male-genital (2018). Accessed 03 May 2019.

14. Grignon D, Brimo F, Comperat E, Delahunt B, Koch M, LopezBeltran A, et al. Urinary tract carcinoma, histopathology reporting guide, biopsy and transurethral resection specimen. 1st edn. Sydney, Australia: International Collaboration on Cancer Reporting (2018). ISBN: 978-1-925687-14-9.

15. International Collaboration on Cancer Reporting. Guidelines for the development of ICCR datasets. http://www.iccr-cancer.org/ datasets/dataset-development (2019). Accessed 03 May 2019.

16. Merlin T, Weston A, Tooher R. Extending an evidence hierarchy to include topics other than treatment: revising the Australian 'levels of evidence'. BMC Med Res Methodol. 2009;9:34.

17. Corbishley C, Chaux A, Colecchia M, Cubilla AL, Shanks J, Velazquez EF, et al. Carcinoma of the penis and distal urethra histopathology reporting guide. 1st edn. Sydney, Australia: International Collaboration on Cancer Reporting; 2017. ISBN: 978-1-925687-05-7.

18. Amin MB, Smith SC, Reuter VE, Epstein JI, Grignon DJ, Hansel DE, et al. Update for the practicing pathologist: the International Consultation on Urologic Disease-European Association of Urology Consultation on bladder cancer. Mod Pathol. 2015;28:612-30.

19. Hansel DE, Amin MB, Comperat E, Cote RJ, Knuchel R, Montironi R, et al. A contemporary update on pathology standards for bladder cancer: transurethral resection and radical cystectomy specimens. Eur Urol. 2013;63:321-32.

20. Chandra A, Griffiths D, McWilliam LJ. Best practice: gross examination and sampling of surgical specimens from the urinary bladder. J Clin Pathol. 2010;63:475-9.

21. College of American Pathologists. Protocol for the examination of specimens from patients with carcinoma of the urinary bladder (2013). http://webapps.cap.org/apps/docs/committees/cancer/ca ncer_protocols/2013/UrinaryBladder_13protocol_3210.pdf. Accessed 03 May 2019.

22. Lopez-Beltran A, Luque RJ, Mazzucchelli R, Scarpelli M, Montironi R. Changes produced in the urothelium by traditional and newer therapeutic procedures for bladder cancer. J Clin Pathol. 2002;55:641-7.

23. Oxley JD, Cottrell AM, Adams S, Gillatt D. Ketamine cystitis as a mimic of carcinoma in situ. Histopathology. 2009;55:705-8.
24. Baker PM, Young RH. Radiation-induced pseudocarcinomatous proliferations of the urinary bladder: a report of 4 cases. Hum Pathol. 2000;31:678-83.

25. Chan TY, Epstein JI. Radiation or chemotherapy cystitis with "pseudocarcinomatous" features. Am J Surg Pathol. 2004;28:909-13.

26. Lopez JI, Schiavo-Lena M, Corominas-Cishek A, Yague A, Bauleth K, Guarch R, et al. Nephrogenic adenoma of the urinary tract: clinical, histological, and immunohistochemical characteristics. Virchows Arch. 2013;463:819-25.

27. Pina-Oviedo S, Shen SS, Truong LD, Ayala AG, Ro JY. Flat pattern of nephrogenic adenoma: previously unrecognized pattern unveiled using PAX2 and PAX8 immunohistochemistry. Mod Pathol. 2013;26:792-8.

28. Paner GP, Ro JY, Wojcik EM, Venkataraman G, Datta MW, Amin MB. Further characterization of the muscle layers and lamina propria of the urinary bladder by systematic histologic mapping: implications for pathologic staging of invasive urothelial carcinoma. Am J Surg Pathol. 2007;31:1420-9.

29. Eble JN, Sauter G, Epstein JI and Sesterhenn IA. World Health Organization Classification of Tumours. Pathology and genetics of tumours of the urinary system and male genital organ. Lyon, France: IARC Press; 2004.

30. Amin MB, Trpkov K, Lopez-Beltran A, Grignon D. Best practices recommendations in the application of immunohistochemistry in the bladder lesions: report from the International Society of Urologic Pathology consensus conference. Am J Surg Pathol. 2014;38:e20-34.

31. Lagwinski N, Thomas A, Stephenson AJ, Campbell S, Hoschar AP, El-Gabry E, et al. Squamous cell carcinoma of the bladder: a clinicopathologic analysis of 45 cases. Am J Surg Pathol. 2007;31:1777-87.

32. Gordetsky J, Epstein JI. Intestinal metaplasia of the bladder with dysplasia: a risk factor for carcinoma? Histopathology. 2015;67:325-30.

33. Gopalan A, Sharp DS, Fine SW, Tickoo SK, Herr HW, Reuter VE, et al. Urachal carcinoma: a clinicopathologic analysis of 24 cases with outcome correlation. Am J Surg Pathol. 2009;33:659-68.

34. Paner GP, McKenney JK, Barkan GA, Yao JL, Frankel WL, Sebo TJ, et al. Immunohistochemical analysis in a morphologic spectrum of urachal epithelial neoplasms: diagnostic implications and pitfalls. Am J Surg Pathol. 2011;35:787-98.

35. Kosem M, Sengul E. Clear cell adenocarcinoma of the urinary bladder. Scand J Urol Nephrol. 2005;39:89-92.

36. al-Izzi MS, Horton LW, Kelleher J, Fawcett D. Malignant transformation in endometriosis of the urinary bladder. Histopathology. 1989;14:191-8.

37. Lah K, Desai D, Hadway P, Perry-Keene J, Coughlin G. Primary vesical clear cell adenocarcinoma arising in endometriosis: a rare case of mullerian origin. Anticancer Res. 2013;33:615-7.

38. Oliva E, Amin MB, Jimenez R, Young RH. Clear cell carcinoma of the urinary bladder: a report and comparison of four tumors of mullerian origin and nine of probable urothelial origin with discussion of histogenesis and diagnostic problems. Am J Surg Pathol. 2002;26:190-7.

39. Sung MT, Zhang S, MacLennan GT, Lopez-Beltran A, Montironi R, Wang M, et al. Histogenesis of clear cell adenocarcinoma in the urinary tract: evidence of urothelial origin. Clin Cancer Res. 2008;14:1947-55.

40. Gilcrease MZ, Delgado R, Vuitch F, Albores-Saavedra J. Clear cell adenocarcinoma and nephrogenic adenoma of the urethra and urinary bladder: a histopathologic and immunohistochemical comparison. Hum Pathol. 1998;29:1451-6. 
41. National Comprehensive Cancer Network (NCCN). NCCN Guidelines (2019). https://www.nccn.org/professionals/physicia n_gls/f_guidelines.asp. Accessed 03 May 2019.

42. Agoff SN, Lamps LW, Philip AT, Amin MB, Schmidt RA, True $\mathrm{LD}$, et al. Thyroid transcription factor- 1 is expressed in extrapulmonary small cell carcinomas but not in other extrapulmonary neuroendocrine tumors. Mod Pathol. 2000;13:238-42.

43. Babjuk M, Burger M, Zigeuner R, Shariat SF, van Rhijn BW, Comperat $\mathrm{E}$, et al. EAU guidelines on non-muscle-invasive urothelial carcinoma of the bladder: update 2013. Eur Urol. 2013;64:639-53.

44. Epstein JI, Amin MB, Reuter VR, Mostofi FK. The World Health Organization/International Society of Urological Pathology consensus classification of urothelial (transitional cell) neoplasms of the urinary bladder. Bladder Consensus Conference Committee. Am J Surg Pathol. 1998;22:1435-48.

45. Pan CC, Chang YH, Chen KK, Yu HJ, Sun CH, Ho DM. Prognostic significance of the 2004 WHO/ISUP classification for prediction of recurrence, progression, and cancer-specific mortality of non-muscle-invasive urothelial tumors of the urinary bladder: a clinicopathologic study of 1,515 cases. Am J Clin Pathol. 2010;133:788-95.

46. Sylvester RJ, van der Meijden AP, Oosterlinck W, Witjes JA, Bouffioux C, Denis L, et al. Predicting recurrence and progression in individual patients with stage Ta T1 bladder cancer using EORTC risk tables: a combined analysis of 2596 patients from seven EORTC trials. Eur Urol. 2006;49:466-465.

47. Herr HW, Donat SM, Reuter VE. Management of low grade papillary bladder tumors. J Urol. 2007;178:1201-5.

48. National Institute for Health and Care Excellence (NICE). NICE bladder cancer guideline. https://www.nice.org.uk/guidance/ng2/ chapter/1-Recommendations\#follow-up-after-treatment-for-nonmuscle-invasive-bladder-cancer-2 (2015). Accessed 03 May 2019.

49. Varma M, Delahunt B, van der Kwast T. Grading noninvasive bladder Cancer: World Health Organisation 1973 or 2004 may be the wrong question. Eur Urol. 2019;76:413-5.

50. The Royal College of Pathologists, UK. Dataset for tumours of the urinary collecting system (renal pelvis, ureter, urinary bladder and urethra). https://www.rcpath.org/uploads/assets/uploaded/ e1c47ffe-756d-4727-88784e509cbde404.pdf (2013). Accessed 03 May 2019.

51. Cao D, Vollmer RT, Luly J, Jain S, Roytman TM, Ferris C, et al. Comparison of 2004 and 1973 World Health Organization grading systems and their relationship to pathologic staging for predicting long-term prognosis in patients with urothelial carcinoma. Urology. 2010;76:593-9.

52. Lopez-Beltran A, Cheng L, Montironi R, Blanca A, Leva M, Roupret M, et al. Clinicopathological characteristics and outcome of nested carcinoma of the urinary bladder. Virchows Arch. 2014;465:199-205.

53. Amin MB, Reuter VE, Epstein JI, Grignon DJ, Hansel DE, Lin $\mathrm{O}$, et al. Pathology Consensus Guidelines by the Pathology of Bladder Cancer Work Group. In: Soloway S, Khoury A, editors. Bladder Cancer. 2nd ed. Paris, France: ICUD-EAU; 2012.

54. Toll AD, Epstein JI. Invasive low-grade papillary urothelial carcinoma: a clinicopathologic analysis of 41 cases. Am J Surg Pathol. 2012;36:1081-6.

55. van Rhijn BW, Musquera M, Liu L, Vis AN, Zuiverloon TC, van Leenders GJ, et al. Molecular and clinical support for a fourtiered grading system for bladder cancer based on the WHO 1973 and 2004 classifications. Mod Pathol. 2015;28:695-705.

56. Patschan O, Sjodahl G, Chebil G, Lovgren K, Lauss M, Gudjonsson $\mathrm{S}$, et al. A molecular pathologic framework for risk stratification of stage T1 urothelial carcinoma. Eur Urol. 2015;68:824-32.
57. Cheng L, Neumann RM, Nehra A, Spotts BE, Weaver AL, Bostwick DG. Cancer heterogeneity and its biologic implications in the grading of urothelial carcinoma. Cancer. 2000;88:1663-70.

58. Billis A, Carvalho RB, Mattos AC, Negretti F, Nogueira CR, Oliveira MC, et al. Tumor grade heterogeneity in urothelial bladder carcinoma-proposal of a system using combined numbers. Scand J Urol Nephrol. 2001;35:275-9.

59. Reis LO, Taheri D, Chaux A, Guner G, Mendoza Rodriguez MA, et al. Significance of a minor high-grade component in a low-grade noninvasive papillary urothelial carcinoma of bladder. Hum Pathol. 2016;47:20-5.

60. Edge SE, Byrd DR, Compton CC, Fritz AG, Greene FL, Trotti A (editors). AJCC cancer staging manual. 7th ed. New York: Springer; 2010.

61. Tosoni I, Wagner U, Sauter G, Egloff M, Knonagel H, Alund G, et al. Clinical significance of interobserver differences in the staging and grading of superficial bladder cancer. BJU Int. 2000;85:48-53.

62. Bol MG, Baak JP, Buhr-Wildhagen S, Kruse AJ, Kjellevold KH, Janssen EA, et al. Reproducibility and prognostic variability of grade and lamina propria invasion in stages Ta, T1 urothelial carcinoma of the bladder. J Urol. 2003;169:1291-4.

63. van Rhijn BW, van der Kwast TH, Kakiashvili DM, Fleshner $\mathrm{NE}$, van der Aa MN, Alkhateeb $\mathrm{S}$, et al. BJU Int. 2010;106:206-11.

64. Compérat E, Egevad L, Lopez-Beltran A, Camparo P, Algaba F, Amin M, Epstein JI, et al. An interobserver reproducibility study on invasiveness of bladder cancer using virtual microscopy and heatmaps. Histopathology. 2013;63:756-66.

65. Ro JY, Ayala AG, el-Naggar A. Muscularis mucosa of urinary bladder. Importance for staging and treatment. Am J Surg Pathol. 1987;11:668-73.

66. Paner GP, Shen SS, Lapetino S, Venkataraman G, Barkan GA, Quek ML, et al. Diagnostic utility of antibody to smoothelin in the distinction of muscularis propria from muscularis mucosae of the urinary bladder: a potential ancillary tool in the pathologic staging of invasive urothelial carcinoma. Am J Surg Pathol. 2009;33:91-8.

67. Paner GP, Brown JG, Lapetino S, Nese N, Gupta R, Shen SS, et al. Diagnostic use of antibody to smoothelin in the recognition of muscularis propria in transurethral resection of urinary bladder tumor (TURBT) specimens. Am J Surg Pathol. 2010;34:792-9.

68. Miyamoto H, Sharma RB, Illei PB, Epstein JI. Pitfalls in the use of smoothelin to identify muscularis propria invasion by urothelial carcinoma. Am J Surg Pathol. 2010;34:418-22.

69. Cheville JC, Dundore PA, Bostwick DG, Lieber MM, Batts KP, Sebo TJ, et al. Transitional cell carcinoma of the prostate: clinicopathologic study of 50 cases. Cancer. 1998;82:703-7.

70. Oliai BR, Kahane H, Epstein JI. A clinicopathologic analysis of urothelial carcinomas diagnosed on prostate needle biopsy. Am J Surg Pathol. 2001;25:794-801.

71. Shen SS, Lerner SP, Muezzinoglu B, Truong LD, Amiel G, Wheeler TM. Prostatic involvement by transitional cell carcinoma in patients with bladder cancer and its prognostic significance. Hum Pathol. 2006;37:726-34.

72. Younes M, Sussman J, True LD. The usefulness of the level of the muscularis mucosae in the staging of invasive transitional cell carcinoma of the urinary bladder. Cancer. 1990;66:543-8.

73. Roupret M, Seisen T, Comperat E, Larre S, Mazerolles C, Gobet $\mathrm{F}$, et al. Prognostic interest in discriminating muscularis mucosa invasion (T1a vs T1b) in nonmuscle invasive bladder carcinoma: French national multicenter study with central pathology review. J Urol. 2013;189:2069-76.

74. Soukup V, Duskova J, Pesl M, Capoun O, Feherova Z, Zamecnik $\mathrm{L}$, et al. The prognostic value of $\mathrm{T} 1$ bladder cancer 
substaging: a single institution retrospective study. Urol Int. 2014;92:150-6.

75. De Marco V, Cerruto MA, D’Elia C, Brunelli M, Otte O, Minja A, et al. Prognostic role of substaging in T1G3 transitional cell carcinoma of the urinary bladder. Mol Clin Oncol. 2014;2:575-80.

76. Orsola A, Werner L, de Torres I, Martin-Doyle W, Raventos CX, Lozano F, et al. Reexamining treatment of high-grade T1 bladder cancer according to depth of lamina propria invasion: a prospective trial of 200 patients. Br J Cancer. 2015;112:468-74.

77. Cheng L, Weaver AL, Neumann RM, Scherer BG, Bostwick DG. Substaging of $\mathrm{T} 1$ bladder carcinoma based on the depth of invasion as measured by micrometer: a new proposal. Cancer. 1999;86:1035-43.

78. Brimo F, Wu C, Zeizafoun N, Tanguay S, Aprikian A, Mansure $\mathrm{JJ}$, et al. Prognostic factors in T1 bladder urothelial carcinoma: the value of recording millimetric depth of invasion, diameter of invasive carcinoma, and muscularis mucosa invasion. Hum Pathol. 2013;44:95-102.

79. van der Aa MN, van Leenders GJ, Steyerberg EW, van Rhijn BW, Jobsis AC. Zwarthoff Ecet al. A new system for substaging pT1 papillary bladder cancer: a prognostic evaluation. Hum Pathol. 2005;36:981-6.

80. Chang WC, Chang YH, Pan CC. Prognostic significance in substaging ofT1 urinary bladder urothelial carcinoma on transurethral resection. Am J Surg Pathol. 2012;36:454-61.

81. van Rhijn BW, van der Kwast TH, Alkhateeb SS, Fleshner NE, van Leenders GJ, Bostrom PJ, et al. A new and highly prognostic system to discern T1 bladder cancer substage. Eur Urol. 2012;61:378-84.

82. Hu Z, Mudaliar K, Quek ML, Paner GP, Barkan GA. Measuring the dimension of invasive component in pT1 urothelial carcinoma in transurethral resection specimens can predict time to recurrence. Ann Diagn Pathol. 2014;18:49-52.

83. Lopez-Beltran A, Bassi PF, Pavone-Macaluso M, Montironi R. Handling and pathology reporting of specimens with carcinoma of the urinary bladder, ureter, and renal pelvis. A joint proposal of the European Society of Uropathology and the Uropathology Working Group. Virchows Arch. 2004;445:103-10.

84. Mariappan P, Finney SM, Head E, Somani BK, Zachou A, Smith $\mathrm{G}$, et al. Good quality white-light transurethral resection of bladder tumours (GQ-WLTURBT) with experienced surgeons performing complete resections and obtaining detrusor muscle reduces early recurrence in new non-muscle-invasive bladder cancer: validation across time and place and recommendation for benchmarking. BJU Int. 2012;09:1666-73.

85. Fritsche HM, Burger M, Svatek RS, Jeldres C, Karakiewicz PI, Novara G, et al. Characteristics and outcomes of patients with clinical T1 grade 3 urothelial carcinoma treated with radical cystectomy: results from an international cohort. Eur Urol. 2010;57:300-9.

86. Shariat SF, Svatek RS, Tilki D, Skinner E, Karakiewicz PI, Capitanio $U$, et al. International validation of the prognostic value of lymphovascular invasion in patients treated with radical cystectomy. BJU Int. 2010;105:1402-12.

87. Eisenberg MS, Boorjian SA, Cheville JC, Thompson RH, Thapa P, Kaushik D, et al. The SPARC score: a multifactorial outcome prediction model for patients undergoing radical cystectomy for bladder cancer. J Urol. 2013;190:2005-10.

88. Kluth LA, Rieken M, Xylinas E, Kent M, Rink M, Roupret M, et al. Gender-specific differences in clinicopathologic outcomes following radical cystectomy: an international multi-institutional study of more than 8000 patients. Eur Urol. 2014;66:913-9.

89. von Rundstedt FC, Mata DA, Groshen S, Stein JP, Skinner DG, Stadler WM, et al. Significance of lymphovascular invasion in organ-confined, node-negative urothelial cancer of the bladder: data from the prospective p53-MVAC trial. BJU Int. 2015;116:44-9.

90. Cha EK, Shariat SF, Kormaksson M, Novara G, Chromecki TF, Scherr DS, et al. Predicting clinical outcomes after radical nephroureterectomy for upper tract urothelial carcinoma. Eur Urol. 2012;61:818-25.

91. Godfrey MS, Badalato GM, Hruby GW, Razmjoo M, McKiernan JM. Prognostic indicators for upper tract urothelial carcinoma after radical nephroureterectomy: the impact of lymphovascular invasion. BJU Int. 2012;110:798-803.

92. Hurel S, Roupret M, Ouzzane A, Rozet F, Xylinas E, Zerbib M, et al. Impact of lymphovascular invasion on oncological outcomes in patients with upper tract urothelial carcinoma after radical nephroureterectomy. BJU Int. 2013;111:1199-207.

93. Lee SM, Russell A, Hellawell G. Predictive value of pretreatment inflammation-based prognostic scores (neutrophil-to-lymphocyte ratio, platelet-to-lymphocyte ratio, and lymphocyte-tomonocyte ratio) for invasive bladder carcinoma. Korean J Urol. 2015;56:749-55.

94. Larsen MP, Steinberg GD, Brendler CB, Epstein JI. Use of Ulex europaeus agglutinin I (UEAI) to distinguish vascular and "pseudovascular" invasion in transitional cell carcinoma of bladder with lamina propria invasion. Mod Pathol. 1990;3:83-8.

95. Lopez JI. Angulo JCThe prognostic significance of vascular invasion in stage T1 bladder cancer. Histopathology. 1995;27:27-33.

96. Kunju LP, You L, Zhang Y, Daignault S, Montie JE, Lee CT. Lymphovascular invasion of urothelial cancer in matched transurethral bladder tumor resection and radical cystectomy specimens. J Urol. 2008;180:1928-32.

97. Andius P, Johansson SL, Holmang S. Prognostic factors in stage T1 bladder cancer: tumor pattern (solid or papillary) and vascular invasion more important than depth of invasion. Urology. 2007;70:758-62.

98. Streeper NM, Simons CM, Konety BR, Muirhead DM, Williams $\mathrm{RD}$, O'Donnell MA, et al. The significance of lymphovascular invasion in transurethral resection of bladder tumour and cystectomy specimens on the survival of patients with urothelial bladder cancer. BJU Int. 2009;103:475-9.

99. Cho KS, Seo HK, Joung JY, Park WS, Ro JY, Han KS, et al. Lymphovascular invasion in transurethral resection specimens as predictor of progression and metastasis in patients with newly diagnosed T1 bladder urothelial cancer. J Urol. 2009; 182:2625-30.

100. Segal R, Yafi FA, Brimo F, Tanguay S, Aprikian A, Kassouf W. Prognostic factors and outcome in patients with $\mathrm{T} 1$ high-grade bladder cancer: can we identify patients for early cystectomy? BJU Int. 2012;109:1026-30.

101. Tilki D, Shariat SF, Lotan Y, Rink M, Karakiewicz PI, Schoenberg MP, et al. Lymphovascular invasion is independently associated with bladder cancer recurrence and survival in patients with final stage $\mathrm{T} 1$ disease and negative lymph nodes after radical cystectomy. BJU Int. 2013;11:1215-21.

102. Branchereau J, Larue S, Vayleux B, Karam G, Bouchot O, Rigaud J. Prognostic value of the lymphovascular invasion in high-grade stage pT1 bladder cancer. Clin Genitourin Cancer. 2013;11:182-8.

103. Olsson H, Hultman P, Rosell J, Jahnson S. Population-based study on prognostic factors for recurrence and progression in primary stage T1 bladder tumours. Scand. J Urol. 2013;47:188-95.

104. Algaba F. Lymphovascular invasion as a prognostic tool for advanced bladder cancer. Curr Opin Urol. 2006;16:367-71.

105. Gardmark T, Bladstrom A, Hellsten S, Malmstrom PU. Analysis of clinical characteristics, management and survival of patients 
with Ta T1 bladder tumours in Sweden between 1997 and 2001. Scand J Urol Nephrol. 2006;40:276-82.

106. Kirkali Z, Chan T, Manoharan M, Algaba F, Busch C, Cheng L, et al. Bladder cancer: epidemiology, staging and grading, and diagnosis. Urology. 2005;66(6 Suppl 1):4-34.

107. Zincke H, Utz DC. Review of Mayo Clinic experience with carcinoma in situ. Urology. 1986;27:288.

108. Wolf H, Olsen PR, Fischer A, Hojgaard K. Urothelial atypia concomitant with primary bladder tumour. Incidence in a consecutive series of 500 unselected patients. Scand J Urol Nephrol. 1987;21:33-8.

109. Koss LG. Mapping of the urinary bladder: its impact on the concepts of bladder cancer. Hum Pathol. 1979;10:533-48.

110. Zieger K, Marcussen N, Borre M, Orntoft TF, Dyrskjot L. Consistent genomic alterations in carcinoma in situ of the urinary bladder confirm the presence of two major pathways in bladder cancer development. Int J Cancer. 2009;125:2095-103.
111. Kulkarni GS, Hakenberg OW, Gschwend JE, Thalmann G, Kassouf W, Kamat A, et al. An updated critical analysis of the treatment strategy for newly diagnosed high-grade T1 (previously T1G3) bladder cancer. Eur Urol. 2010;57:60-70.

112. Dyrskjot L, Zieger K, Real FX, Malats N, Carrato A, Hurst C, et al. Gene expression signatures predict outcome in non-muscleinvasive bladder carcinoma: a multicenter validation study. Clin Cancer Res. 2007;13:3545-51.

113. Choi W, Porten S, Kim S, Willis D, Plimack ER, HoffmanCensits $\mathrm{J}$, et al. Identification of distinct basal and luminal subtypes of muscle-invasive bladder cancer with different sensitivities to frontline chemotherapy. Cancer Cell. 2014;25:152-65.

114. Srigley JR, McGowan T, MacLean A, Raby M, Kramer RossJ, et al. Standardized synoptic cancer pathology reporting: a population-based approach. J Surg Oncol. 2009;99:517-24. 